\title{
Plexiform schwannoma arising from the duodenum: a rare condition
}

\begin{abstract}
Summary
Plexiform schwannoma is a benign tumor originated in the sheath of the peripheral nerves that is composed of Schwann cells that are arranged in palisade. These lesions usually arise from the skin being less frequent in intraabdominal organs. We describe, according to the reviewed literature, the second case of plexiform schwannoma arising from the duodenum. A 49 year old patient with a history of vomiting, diffuse abdominal discomfort and weight loss. The complementary tests were inconclusive. The patient was operated evidencing in the operation room a stenosis of the third duodenal portion without any other alterations. The pathological anatomy reported a submucosal lesion formed by multiple nodules composed by a proliferation of spindle cells that were positive for S-100.
\end{abstract}

Keywords: duodenum, plexiform scwannoma, inmunohistochemistry
Volume 9 Issue 2 - 2018

\author{
Rosa Martí Fernández,' Marina Garcés,' \\ Roberto Martí,' Claudia Mestre, ${ }^{2}$ Joaquín \\ Ortega' \\ 'Department of General Surgery, Hospital Clínico, Universitario \\ of Valencia, Spain \\ ${ }^{2}$ Department of Pathology, Hospital Clínico, Universitario of \\ Valencia, Spain
}

Correspondence: Rosa Martí Fernández, Correo electrónico, Avenida Blasco lbáñez number 17, CP 46010,Valencia, Spain, Tel 961973500,Email rosamartifernandez@gmail.com

Received: January 18, 2018 | Published: March 09, 2018
Abbreviations: CT, axial computed tomography; MRI, magnetic resonance image; GIST, gastrointestinal stromal tumors; MEA, membrane epithelial antigen

\section{Introduction}

Plexiform schwannoma is a tumor with benign behavior originated in the sheath of peripheral nerves composed of Schwann cells that are arranged in palisade. ${ }^{1-5}$ It is considered an unusual variant, with an approximate incidence of $4-5 \%$ of all schwannomas. They are usually well-defined, localized lesions on the skin or the subcutaneous tissue and settle in decreasing order in the head and neck, upper and lower limbs. Intraabdominal localization is less frequent, with a few cases published in the literature. There is only one case published of duodenal plexiform schwannoma. ${ }^{4}$ With the following communication we present the second case of duodenal plexiform schwannoma.

\section{Presentation of the case}

A 49-year-old male with no medical history of interest who consults to the emergency department in several occasions for weight loss $(7 \mathrm{~kg})$, postprandrial vomiting, and epigastric pain of a month of evolution.

After performing a high endoscopy a stenosis of the second duodenal portion without any underlying cause was seen. Subsequently an echoendoscopy was performed in which it was appreciated a stenosis at the same zone, with a thickening of the wall up to $7 \mathrm{~mm}$, at the expense of the mucosa and submucosa. Diffuse hypoechogenic areas with significant stiffness were observed without allowing the echoendoscope to progress. The pathological anatomy samples taken in these tests were not conclusive and reported non-specific duodenitis. The results of the blood tests showed no pathological findings.

The CT scan with oral contrast showed a dilatation of the first and second duodenal portion and a stenosis in the zone of the duodenal knee, between the second and third duodenal portion (Figure 1). The MRI presented a prominent pancreatic gland with enhancement after contrast administration without appreciation of masses. Regional lymph nodes were not affected.

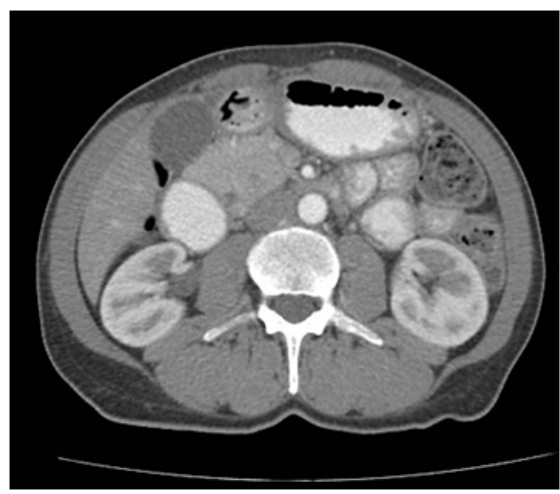

Figure $1 \mathrm{CT}$ scan showing duodenal stenosis.

Given the clinical deterioration while the study was completed and the absence of a certainty diagnosis, surgical treatment was carried out. The patient was operated by a bilateral subcostal laparotomy. Intraoperatively, a circular stenosis was observed in the third duodenal portion that caused marked proximal dilatation of the duodenum. No lymphadenopathies or other lesions at the abdominal cavity were found. Duodenum was sectioned beneath Vater's papilla, after its identification, practicing cholecystectomy after tutoring the common bile duct, and the resection was extended to the jejunum $(5 \mathrm{~cm}$ below the Treitz angle). Practicing, therefore, a duodenectomy of the third and forth portion. The transit was restored by an anastomosis duodenojejunal side to side to the anterior face of the second duodenal portion. The patient was discharged at the fifth day without any complications.

The histological study of the piece revealed the presence of a submucosal lesion composed by numerous nodules surrounded by a thin fibrous capsule and conformed by a proliferation of spindle cells. In the inmunohistoquimic analysis this cells were positive for S-100, thus confirming the diagnosis of plexiform schwannoma (Figure 2) 
(Figure 3). The sample presented two lymph nodes without data of malignancy.

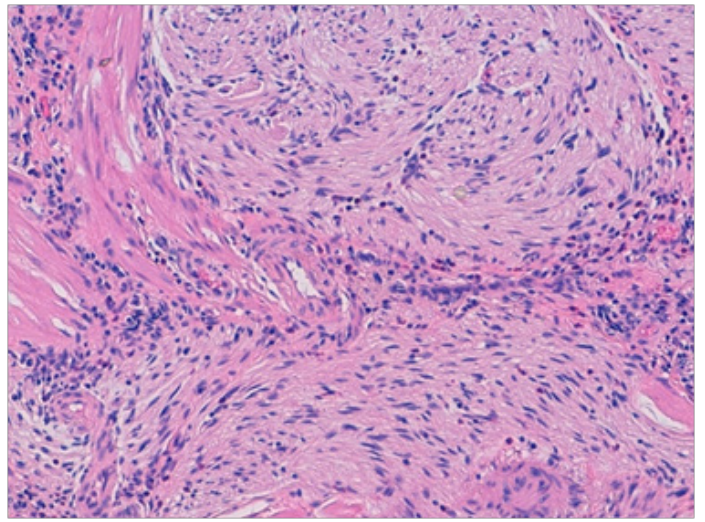

Figure 2 Palisade arrangement of the nuclei in areas of higher nuclear density can be appreciated. Hypocellular areas present a fibrillar appearance.

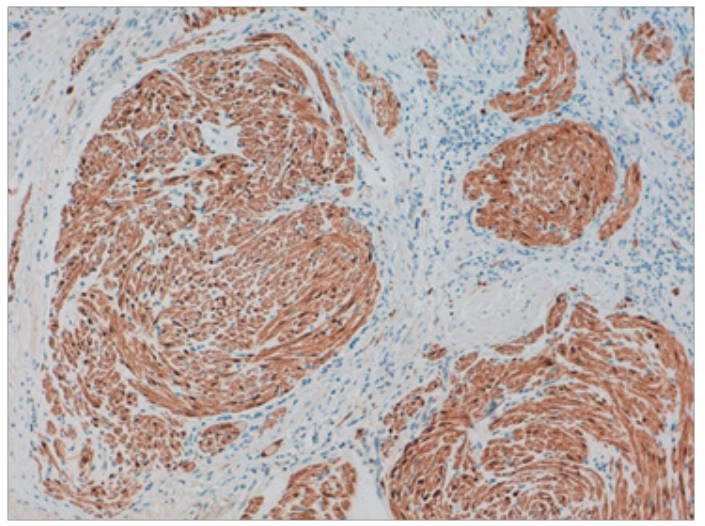

Figure 3 Staining for S-100 positive.

\section{Discussion}

Schwannomas are considered a rare variant of mesenchymal tumors of the digestive system, with an approximate incidence of $2-3 \%$ of all mesenchymal tumors; the most common of ones are gastrointestinal stromal tumors (GIST). There are numerous variants of schwannoma, such as: cellular, epitheliod, plexiform, glandular or melanocytic, among others; being the plexiform variant infrequent, with an estimated incidence of approximately $5 \%$ of all schwannomas. ${ }^{1,3,5}$

The plefixorm variant use to be unique lesions with a variable size. There is no predilection for any sex. The most frequent is that the settle on soft tissues, especially, in the head and neck. Its abdominal presentation is rare. Among the most commonly affected intrabdominal organs are the esophagus and the colon, and only one case of plexiform schwannoma located in the duodenum has been described in the reviewed literature. ${ }^{1,5}$

These are tumors with little clinical expression that occur with vague symptoms as abdominal discomfort, intermittent episodes of intestinal bleeding or, more rarely, intestinal obstruction. In our case, the patient debuted with a clinic of high intestinal obstruction with postprandial vomiting, epigastric pain and weight loss.

Its multiple presentation in patients in the first or second decade of life may make us suspect the presence of an underlying syndrome, such as neurofibromatosis type 2 or schwannomatosis, being more frequent the association with the neurofibromatosis type 2 . Both entities are genetic disorders that are inherited with autosomic dominant character and are characterized by the development of multiple tumors (neurofibromas and schwannomas) in different anatomic localizations. Nevertheless, it is important to mention that the association with these is rare, with few cases in which both conditions coexist. ${ }^{3}$

The histological characteristics of plexiform schwannoma are the presence of multiple nodules composed by fusiform cells generally delimited by a thin fibrous capsule, which delimits well the lesion. They are composed by Schwann cells. These lesions are characterized by being hypercellular. The hypercellular areas with disposition of cells in palisade are known as Antoni A and, sometimes, we can appreciate the formation of the typical bodies of Verocay. The presente of hipocellular areas or Antoni B is less frequent. Degenerative changes such as intratumoral necrosis, mithosis or intratumoral hemorrhage are uncommon. It is characteristic that in almost all of studies of immunohistochemistry of this type of tumor they result strongly and diffusely positive for S-100 (which indicates the presence of Schwann cells), while they are negative for another markers such as C-kit (CD 117), desmin or smooth muscle actin, vimentin, which are characteristic of other mesenchymal tumors such as GIST, leiomyomas or schwannomas respectively. ${ }^{3,5}$

Differential diagnosis should be made with other mesenchymal tumors such as GIST, leiomyomas and plexiform neurofibroma. In these cases, the immunohistochemical analysis of the pieces is essential. GISTs have positive reaction for c-kit protein, leiomyomas for desmin and smooth muscle actin and plexiform neurofibroma is positive for S-100, CD34 and less frequently MEA. While they are not positive for S-100, with the exception of plexiform neurofibroma, being one of the main diagnostic features of plexiform schwannoma, which they react with this protein in almost all cases. Data obtained through imaging tests, mainly CT, may be helpful although there are no pathognomonic images for plexiform schwannoma. They behave as a well defined and shaped mass, homogeneous, without contrast uptake after the administration of the same. These facts can be differentiated from GIST, which is seen in the imaging tests as a heterogeneous mass, with frequent degenerative changes such as necrosis, intratumoral hemorrhage or cystic changes (absent in schwannoma plexiform). It is essential to perform the differential diagnosis with plexiform neurofibroma, tumors that appear in neurofibromatosis type I or Von Recklinghausen disease, given its potential for malignization. In imaging tests, nuclear magnetic resonance imaging (MRI), these tumors appear as well delimited isointense or slightly hyperintense lesions in T1-phase, with enhancement in form of target in T2 sequence. While the plexiform schwannoma in MRI is seen as hypointense lesions in T1 sequence and hyperintense in T2 sequence. ${ }^{6,7}$

The treatment of this type of tumors is surgical excision with excellent results if complete excision is achieved. They are benign, non-infiltrating tumors with limited or no local recurrence and no ability to metastasize to other organs, so the prognosis is favorable. . $^{1,4,8,9}$

In conclusion, plexiform schwannoma is a rare entity that tends to settle in soft tissues of the head and neck, being infrequent visceral involvement. These are well-defined single lesions with marked hypercellularity and positive for S-100. Its association with 
neurofibromatosis type 2 or schwannomatosis is rare. Treatment consists in surgical resection with good results and good prognosis. ${ }^{10}$

\section{Acknowledgements}

Thanks to the service of pathological anatomy for the transfer of images.

\section{Conflict of interest}

The authors declares no conflict of interest with the publication of the article.

\section{References}

1. Aktekin A, Özkara S, Meriç K, et al. Plexiform schwannoma of the duodenum accompanying pyloric stenosis: Report of a case. Turk $J$ Gastroenterol. 2012;23:885-889.

2. Iida A, Imamura $\mathrm{Y}$, Katayama K, et al. Plexiform Schwannoma of the small intestine: report of a case. Surg Today. 2003;33:940-943.

3. Kudose S, Kyriakos M, Magdi Awad M. Gastric plexiform schwannoma in association with neurofibromatosis type 2. Clin J Gastroenterol. 2016:9:352-357.
4. Nagata M, Ito H, Matsuzaki T, Furumoto H, et al. Plexiform schwannoma involving the trachea and recurrent laryngeal nerve: a case report. Surg Case Re. 2015;1:67.

5. Chikkannaiah PM. Boovalli M, Nathiyal M, et al. Morphological spectrum of peripheral nerve sheath tumors: An insight into World Health Organization 2013 classification. J Neurosci Rural Pract. 2016;7(3):346-54

6. Levy AD, Quiles AM, Miettinen M, et al. Gastrointestinal Schwannomas: CT Features with Clinicopathologic Correlation. AJR 2005;184:797802

7. Carranza A, Salinas MV, Ávila R, et al. Problemas diagnósticos en tumores del nervio periférico (I). Rev Esp Patol. 2011;44(2):97-116.

8. Dhua. A rare case of plexiform schwannoma of the lower lip: Treatment and management. Indian J Plast Surg. 2015;48(2):208-211.

9. Alcaraz-Mateos E, Rojo-España R, Pérez-Ramos M, et al. Schwannoma epitelioide con cambios tipo ancient. A propósito de un caso. Rev Esp Patol. 2014;47(2):114-117.

10. Batalla A, De la Torre C, Dávila P, et al. Schwannoma plexiforme: presentación de un caso y diagnóstico diferencial histopatológico. Med Cutan Iber Lat Am. 2013;41(3):122-125. 